\title{
Dos modelos en el diagnóstico y tratamiento de las dificultades de aprendizaje
}

S. MAGENDZO * y A. MAGENDZO **

\section{Introducción a los Modelos}

La atención de los niños que presentan dificultades de aprendizaje constituye uno de los desafíos más importantes que enfrenta nuestra educación. Para nadie es un misterio el alto porcentaje de alumnos que año a año, fracasa, sc frustra y deserta, con la consecuente repercusión psicológica que esto tiene para el individuo y su familia y el gravamen económico que esto significa para la sociedad.

La solución de este problema es un imperativo social insoslayable que debe ser considerado como prioritario en todo programa de acción educativa.

Al respecto, se pueden mencionar dos grandes líneas de solución que, a pesar de complementarse, difieren en su enfoque práctico.

Un primer modelo enfatiza la idea que existen diferencias inter e intra individuales en el desarrollo de las conductas escolares. En otras palabras, se postula que cada individuo, además de las diferencias que presenta en los diversos planos conductuales de su persona, presenta también algunas que lo distinguen de sus congéneres. Este Modelo intenta medir estas diferencias tratando de identificar los procesos que las causan. Vale decir, se apunta al diagnóstico de las habilidades que subyacen a las conductas escolares a través de tests que supuestamente son válidos y confiables y que tienen una estandarización normativa.

En este Modelo se tiende, consecuentemente, a usar prucbas de tipo general, verbigracia: el Test Visor Motor de Bender, el Test de Desarrollo

\footnotetext{
* Del Hospital Luis Calvo Mackenna, Depto. de Neurulogla Y Psiquiatría y del Programa Interdisciplinario de Investigactioties en Educación de Ja Universidad Cat 6 lica de Clilic.

* Del Programa Inlerdicciplinario de lnvestigaciones en Educación (PIlE) de la Universidad Católica de Chile.
}

Perceptual de Frostig, el Test de Habilidades Psicolingüísticas de Kirk, el Test de Inteligencia de Wechler, etc. Los resultados que arrojan estos tests permiten descubrir un perfil de habilidades en el alumno. Perfil que orienta Ia enseñanza diferenciada y el tratamiento psicopedagógico.

Tomemos el Test de Desarrollo Perceptual de Frostig, con el fin de ilustrar este Modelo. En él se evalúan cinco aspectos relacionados con la percepción: coordinación visomotora percepción, figura y fondo, constancia perceptiva, percepción de posición, percepción de relaciones espaciales.

Los resultados que arroja cada uno de estos aspectos del Test de Desarrollo son graficados en un "Perfil" que señala con precisión el grado de dificultad que el alumno presenta. Además, como el test es normativo posibilita la ubicación del alumno con respecto al grupo al cual pertenece.

Ahora bien, a la luz de las alteraciones que el alumno presente frente a esta prueba se orientará Ia rehabilitación escolar. Los autores de! test postulan que el tratamiento de estas habilidades subyacentes repercutirá favorablemente en el rendimiento de las conductas escolares como lectura y escritura. Esta posición se ve respaldada por las correlaciones encontradas entre las habilidades perceptivas, medidas por el Test de Frostig y el rendimiento en lectura y escritura.

El segundo enfoque o Modelo se centra preferentemente en las habilidades específicas del curriculum. Estas habilidades se encuentran en una secuencia ordenada de objetivos, que ha sido preparada acorde a un minucioso análisis de tarens. Es decir, cada materia escolar está desglosada en pasos ordenados y lógicos que permiten el logro de cicrtos objetivos terminales.

Disponiendo de esta jcrarquización, los que postulan por este Modelo señalan que el diagnóstico de las dificultades de aprendizaje se realiza 
a través de la ubicación del alumno cn dicha secuencia.

En el ejenplo que se acompaña, se ilustra la secuencia educacional con respecto a un objetivo terminal en el área de la discriminación auditiva.

\section{Objetivo Terminal}

1.- "Dado, en forma oral, un conjunto de palabras, el alumno identificará aquéllas que riman" (gato, peras, pato, garra, etc.).

\section{Objetivos en Ruta}

1.1.- Dado un conjunto de palabras tcrminadas con vocales, el a'umno identificará aquéllas que riman (saco, mano, pala, pare).

1.2.- Dado un conjunto de palabras terminadas en consonantes, el alumno identificará aquéllas que riman (árbol, pape!, sacar, casas).

1.3.- Dado un conjunto de palabras el altumno indicará aquéllas que riman en sus consonantes intermedias (cabeza, morena, pelota, cubierta).

1.4.-- Dado uл conjunte de palabras el alumno indicará aquéllas que riman en sus vocales intermedias (zapato, colado, pisito).

1.5.--Dado un conjunto de palabras el alumno identificará aquéllas que riman en sus consonantes iniciales (lámina, página, lámpata).

1.6.- Dado un conjunto de palabras, el alumno identificará aquéllas que riman en sus vocales iniciales (arte, antes, estufa, uno).

1.7.-Dado un conjunto de palabras, el alumno identificurá aquéllas que son iguales (casa, mesa, cara, casa).

1.8. - Dado un conjunto de letras, el alumno identificará aquéllas que son iguales.

1.9. - Dado un conjunto de sonidos el alumno identificará aquéllos que son iguales.

Por medio de pruebas relacionadas con cada paso de la secuencia (criterios de referencia) se realizará el diagnóstico de la discriminación auditiva. Lo que se desea saber es la ubicación en que se encuentra el alumno en la secuencia educacional orientada hacia el objetivo terminal (Proger 1971, Mann 1972).

Supongamos que el alumno tiene dificultades en el objetivo 1.4: identificación de vocales intermedias; el tratamiento entonces comenzará ejercitando este paso para luego rchabilitar los siguientes objetivos en ruta hasta que el alumno logre aprender el objetivo terminal.

\section{Análisis comparativo}

Considerando to anteriormente expuesto, queda de manifiesto que el primer enfoque apunta a aquellas habilidades o procesos subyacentes, que, supuestamente, son los que determinan las dificultades que el alumno enfrenta. Consecuentemente, se centra en procesos generales, distanciándose de los objetivos más específicos de la cnseñanza. Esto determina que las normas de rchabilitación se hagan difíciles de precisar. Una de las objeciones que se puede hacer a este enfoque cs que no cxiste una confirmación empírica que permita sostener claramente que la rehabilitación de las habilidades generales es un pre-requisito para la adquisición de los objetivos que la enseñanza se plantea (Cohen 1969).

$E_{n}$ el enfoque que hace hincapié en el análisis de tareas se rechaza la idea de habilidades o perfiles de procesos con el fin de inferir dificultades en el aprendizaje. Lo que se pretende es analizar cuáles son los objetivos pre-requisitos para alcanzar un objetivo terminal. Tanto el diagnóstico, como la rehabilitación se centran en las conductas espccíficats que se desean alcanzar a través de un proceso de enseñanza. En otras palabras, e] profesor o psicólogo no se pregunta por las habilidades generales, ni trata de que el alumno las alcance como condición para el aprendizaje, sino que ubical al alumno en una secuencia de aprendizajes y determina qué es lo que el estudiante debe ser capaz de hacer para lograr el dominio de una tarea compleja.

Ambos enfoques dificren, también, en los instrumentos que se cmplean para el diagnóstico.

En el primer caso, se utilizan las pruebas normativas, $y$ en el segundo, criterios de referencia. Tal como veíamos anteriormente, las pruebas normativas, tienden a ubicar al individuo con respecto a determinadas habilidades en relación a un srupo; en cambio cuando se emplean pruebas basadas en criterios de referencia se construyen instrumentos que determinan la posición de un individuo con respecto a una jerarquía de aprendizaje conducentes al logro de un objetivo terminal. El instrumento está formado por ítems representativos de los aprendizajes que faciliten la adquisición de la tarea u objetivo terminal. En el ejemplo de la discriminación auditiva los subobjetivos intermedios orientan la construcción de pruebas diagnósticas.

Siempre que hablamos de un instrumento de medición, uno debe cuestionar el nivel de confiabilidad y validez de éstos, ya que según los resultados obtenidos en las pruebas se toman decisiones educacionales importantes.

Las pruebas normativas requieren una confiabilidad del 0.90 como nivel mínimo aceptable. Es interesante acotar que muy pocos de estos instrumentos alcanza dicho nivel de confiabilidad. 
En las pruebas basadas en criterios de referencia - por ser congruentes con los objetivos de la jerarquía- su validez y confiabilidad son más fáciles de lograr.

Es importante destacar, que en ambos modelos se deben considerar algunos factores de interferencia que pueden influir en la adquisición de ciertas conductas de aprendizaje, verbigracia, factores emocionales, visuales, auditivos, etc. Sin embargo, se puede establecer que existen diferencias de enfoque entre ambos Modelos con respecto a los factores de interferencia.

En el Modelo normativo los factores interferentes son tratados, por lo general, en forma independicnte al problema de aprendizaje. El niño que, por ejemplo, presenta junto a las dificultades lectoras un trastorno emocional, es referido para el tratamiento de éste a un especialista.

En el Modelo de Análisis de Tareas al tratar el problema de aprendizaje se organiza el ambiente de enseñanza, de manera que considere los factores interferentes. En el tratamiento de las dificultades lectoras se planifican los ejercicios tcniendo muy en cuenta, las conductas emocionales del niño.

Más aún, en este Modelo la jerarquía de objetivos se irá modificando $\mathrm{y}$ adaptando a la luz de los factores interferentes que se presentan.

\section{Implicancias para médicos}

$y$ otros especialistas

La aplicación del Modelo de Análisis de Tareas está indicando que el maestro puede ayu- dar enormemente en el diagnóstico y tratamienso de los trastornos de aprendizaje. Los Programas de Estudios están haciendo cada vez más hincapié en la formulación y jerarquización de los objetivos educacionales y en los instrumentos de evaluación; elementos que indiscutiblemente facilitan al profesor la detectación y rehabilitación de dificultades de aprendizaje.

El médico y el psicólogo que muchas veces están en contacto con el maestro pueden insinuar y exigir que éste haga uso de las guías curriculares para definir con mayor precisión los problemas escolares de sus alumnos.

De esta manera, el médico podría determinar a la Juz del informe más preciso del maestro la conducta a seguir frente a las preguntas que los padres les hacen sobre los problemas de sus hijos.

\section{REFERENCIAS}

Colien, S.A. Studies in Visual Perception and Reading in Disadvantaged Children: Journal of Learning Disabilitics, 1969, 2, 498-503.

Magendzo, A. Análisis de Tareas o Jerarquización de Aprendizaje, CPEIP, 1970.

Mann, Lester. The Ground Game in Special Education: Journal of Special Education 1971, 5, 53-65.

Proger, Berton, 8. Program Evaluation: The Model-Building Game. Journal of Letrning Disabilities, 1971, 4 (6) 292-306. 